\title{
Motion of granular particles on the wall of a model silo and the associated wall vibrations
}

\author{
J. M. Buick $\uparrow £$, J. Y. Ooi $\ddagger$, Pankaj $\ddagger$ J. Chavez-Sagarnaga $\ddagger$, A. \\ Pearcełand G. Houghton $\ddagger$ \\ $\dagger$ Physics and Electronics, School of Biological, Biomedical and Molecular Sciences, \\ The University of New England, Armidale, NSW 2351, Australia. \\ $\ddagger$ Institute for Infrastructure \& Environment, School of Engineering \& Electronics, \\ University of Edinburgh, Edinburgh EH9 3JN, U.K.
}

\begin{abstract}
Dynamic phenomena during discharge of a silo are not well understood. This study uses a dual approach to quantify the motion of both the particulate solid at the wall of a model silo and the wall itself. The motion of particles was recorded visually using a digital CCD camera. The particles are seen to move with an intermittent motion. Image analysis techniques were then applied to obtain quantitative measurements of the particulate velocity. Related to the particle motion, the silo walls were observed to vibrate during discharge. These vibrations were measured using a laser vibrometer to obtain independent measurements of the radial displacement and velocity. This enabled comparisons to be made between the particulate motion and the wall vibrations. Results are presented for barley and for PET pellets.
\end{abstract}

Submitted to: J. Phys. D: Appl. Phys.

$\S$ To whom correspondence should be addressed (jbuick@une.edu.au) 


\section{Introduction}

Wall vibrations in full scale industrial silos have been investigated recently by a number of authors and several sources of dynamic excitation in silos have been proposed $[1,2,3,4,5,6,7,8,9]$. These include dilation (density changes) in the solid during flow; stick-slip behaviour between stored solids and silo walls; internal stick-slip behaviour within stored solids; alternating flow patterns during flow; and intermittent collapsing arches in the solid. Although in some studies (e.g. [10, 11]) silo honking (or music) and silo quaking (or shocks) are considered together, these are two distinct phenomena. Industrial experience shows that silos may both quake and honk, may quake but not honk or may honk but not quake. Silo honking is a high frequency sound, similar to a very loud truck horn, that emanates intermittently from a silo during discharge. While silo honking leads to severe noise pollution, there is little concern that it may cause structural integrity problems. Studies conducted by Buick et al. [8, 9] have shown that honking results from a silo structure vibrating as a giant speaker at high frequencies (a few hundred Hertz) in a fixed harmonic set of frequencies (a fundamental acoustic frequency and its multiples). On the other hand, quaking has a predominantly low frequency and a high displacement amplitude response that can be defined as band limited low frequency noise. Industrial silos have been known to fail due to quaking. The excitation mechanism is not fully understood for either quaking or honking however, it has been suggested $[3,12]$ that stick-slip motion of the particulate fill against the silo walls plays an important role in these dynamic phenomena. Indeed it has been shown that polyethylene terephthalate (PET) particles that are known to honk exhibit stick-slip behaviour against alumininium plates while polypropelene pellets that are not known to honk do not $[9,13]$. This paper presents measurements of the wall vibrations that were taken during discharge of a model silo with transparent polycarbonate walls. The motion of the particles against the wall were recorded digitally and analysed to provide quantitative information on particle motion. The relationship between particle motion and wall vibration is discussed.

\section{Experimental procedure}

The experimental system is shown in figure 1. The model silo was $1700 \mathrm{~mm}$ high with a diameter of $650 \mathrm{~mm}$ and was constructed from $1 \mathrm{~mm}$ thick transparent Lexan polycarbonate. The silo had a flat bottom and discharged through a central hole with a diameter of $65 \mathrm{~mm}$. The silo was filled to a height of between 1100 and $1300 \mathrm{~mm}$ from a holding box above the silo, which was filled from the silo discharge using an auger. Both the auger and the refill from the holding box were stopped during the period while the measurements were taken. This was to ensure that the observed vibration was a result of the silo discharge and was not influenced by the preceding filling process. It is also important to ensure that vibrations do not arise from stopping the fill process. Therefore sufficient time was allowed for any such vibrations to decay before taking 


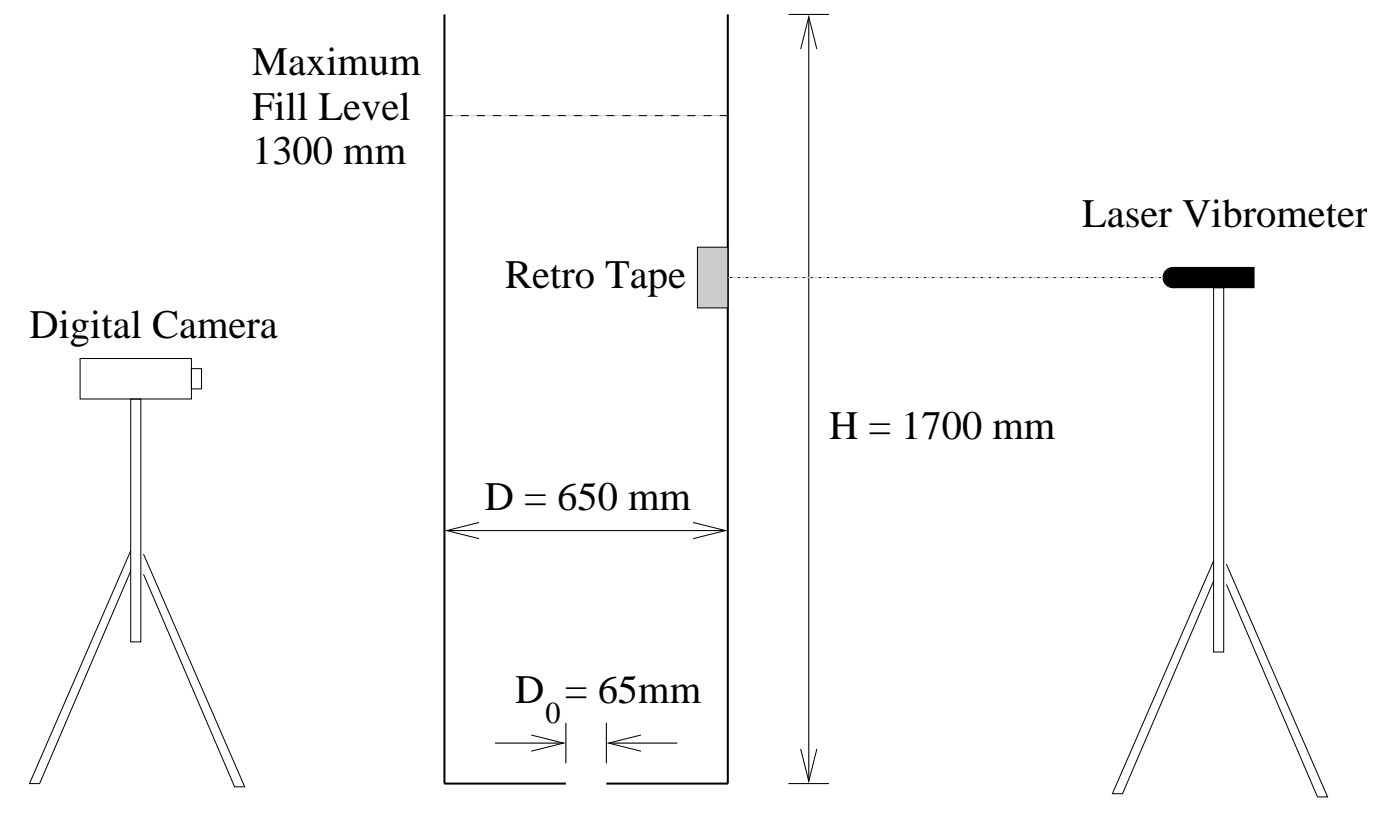

Figure 1. Experimental set-up.

any measurements. Silo wall vibrations were measured using a Polytec laser vibrometer OFV-3001-23/OFV-303 which was directed along the radial direction of the silo onto a piece of reflective retro tape on the silo wall. The retro tape was used to reflect the laser beam enabling a high quality reflection to be obtained from a transparent material. The laser vibrometer was used to measure the radial velocity and displacement of the wall. The velocity is calculated from the Doppler shift of the reflected light, while the displacement is determined from the phase of the light which is circularly polarised. This gives two independent measurements of the wall vibrations. Images of the particles moving against the model silo wall were also obtained using a Sony DCR-TRV340E digital camera. These images were analysed to obtain quantitative information about the particle velocity at the wall.

Two different particulate solids were used for the experimental studies: PET and barley. PET pellets considered were in the shape of flattened cylinder with an elliptic cross-section. The typical cross-section of these pellets was $4 \times 1.5-2 \mathrm{~mm}$ with $4 \mathrm{~mm}$ height and density was in the range $770-890 \mathrm{~kg} / \mathrm{m}^{3}$. The barley had a density in the range $714-867 \mathrm{~kg} / \mathrm{m}^{3}$ and had a typical length of approximately $10 \mathrm{~mm}$.

\section{Analysis of particle images}

The motion of the granular particles against the model silo wall was observed to be intermittent for both PET and barley. The particles remained stationary against the silo wall for a short period of time. After this the particles moved downwards in a short burst of motion before becoming stationary again. The time scales for the stationary and the moving phases were different for PET and for barley. This motion was captured 


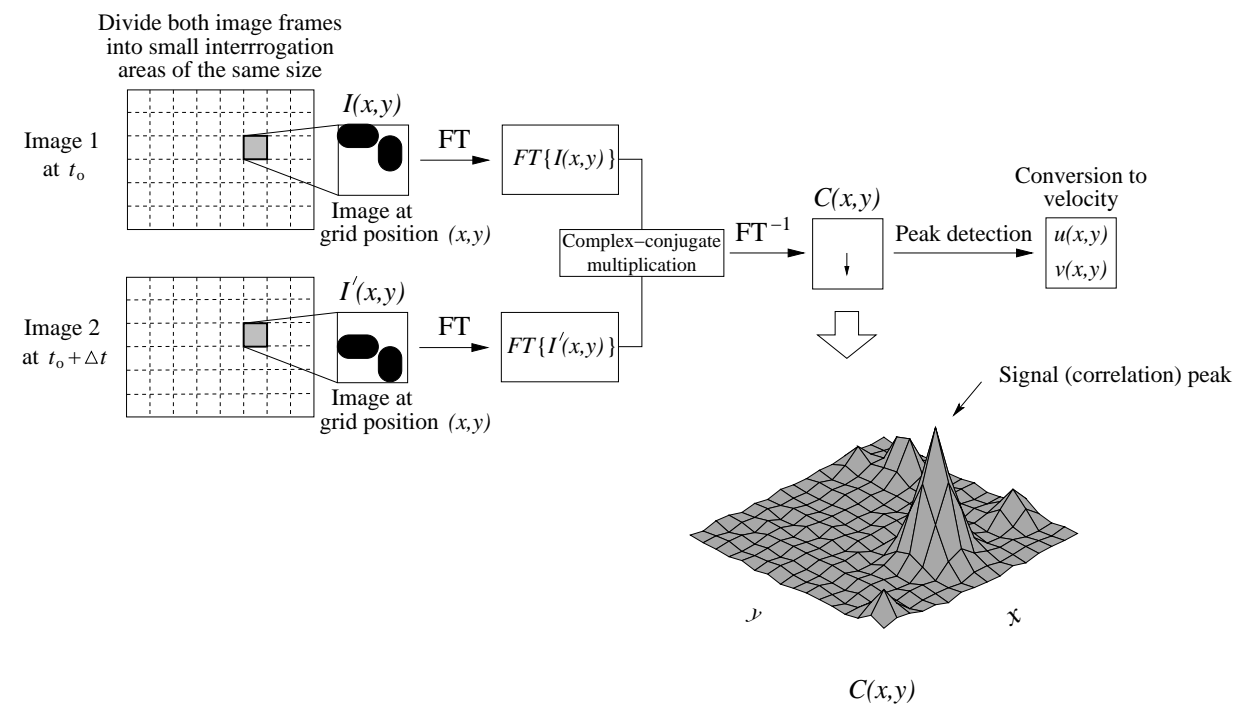

Figure 2. Analysis of the digital images to obtain velocity information.

using a digital camera. This enabled the motion to be viewed and qualitatively assessed as above. To quantatively investigate the particle motion, the successive digital images were analysed using the cross-correlation technique which is a well established approach to determining the velocity profile of small tracer particles in particle image velocimetry $[14,15]$ and has also been shown to be applicable to larger-scale objects [16]. The approach is summarised in figure 2 .

Two images taken at a small time separation $\Delta t$ are considered. Each image is divided into small interrogation areas of the same size. In this study regions 32 by 32 pixels were used. Each interrogation region consists of an intensity map, $I$, over a small region of space. In figure 2, the selected regions show two particles which are clearly seen to move between the images. The two intensity maps are then Fourier transformed using an FFT and the cross-correlation performed in Fourier space. The correlation plane, $C$, is then obtained through an inverse transform. The position of the peak in the correlation plane, see figure 2, corresponds to the mean displacement between the two intensity maps. The position of the peak in the correlation plane is determined to sub-pixel accuracy using a Gaussian peak fitting algorithm. Finally, the pixel displacement is converted to a velocity vector using the magnification of the image and the time separation of the frames. The magnification, measured in pixels per mm, was obtained by taking an image of a metal ruler against the silo wall, while the time separation of the images is determined by the frame rate of the camera. Applying this technique in each interrogation region in the image gives a two-dimensional vector map of the particle velocity. Each vector in the map corresponds to the average velocity within the interrogation region and the velocity vectors lie on a regular grid. This analysis gives the mean translational velocity of the particles, no information concerning 
particle rotation can be obtained. Two examples are shown in figures 3 and 4 for PET and barley particles respectively. In figure 3, the PET particles are of a similar size to the interrogation region, thus each velocity vector corresponds approximately to the translational motion of a single particle. Comparing figures 3(a) and 3(b) the motion of the individual PET particles can be clearly seen between the images (for example see particles marked A and B) and appears to be uniform over the whole image. The vector map in figure 3(c) confirms this. This shows an approximately constant velocity over the whole of the image with a velocity of around $5 \mathrm{~mm} / \mathrm{s}$ at that instant. In figure 3(c) there are a number of missing vectors. These arise from outliers which occur when there is no significant peak in the correlation plane. This can occur, for example, when a single particle fills one of the interrogation regions giving very poor contrast between each of the pixels. There is also a slight variation in the magnitude of the vectors in the bottom row of figure 3(c), this may be due to a slight distortion of the image at the bottom edge. All the vectors in figure 3(c) are pointing in the negative $z$ direction indicating that there is negligible horizontal motion of the particles. The time between successive images was $0.12 \mathrm{~s}$; in figure 3 the separation between (a) and (b) is $3 \Delta t$ to highlight the motion of the PET particles.

The images in figure 4 show a larger region of the silo wall with barley as the fill particles. In this case there are several particles in each image and each vector in figure 4(c) corresponds to the average velocity in the interrogation region. In figures 4(a) and 4(b) there is a considerable amount of glare in the left-hand half of both images. It proved to be difficult to illuminate a large region of the silo sufficiently to obtain good quality images without observing some reflection of the light in the image. The glare on the left-hand half of figures 4(a) and 4(b) render this part of the image unusable for obtaining quantitative velocity information. However, given the curved nature of the silo wall, it was not possible to focus the camera across the variation in the depth of field across the image. For this reason the camera was focused on the region in the right-hand half of figures $4(\mathrm{a})$ and $4(\mathrm{~b})$ and it was therefore not necessary to investigate alternative forms of lighting. This means that the vectors obtained in the left-hand half of figure 4(c) are unreliable and only the vectors in the right-hand half of the image can be considered. In this larger region the vectors are still very regular. This was observed in all vector maps for both sets of images and suggests that the temporal variation in the velocity can be investigated by considering the spatially averaged velocity over the whole of the image for which meaningful velocity information was obtained. That is, in images such as those shown in figure 3 the whole vector field is used in the spatial averaging, while for images such as those shown in figure 4 we only use the region where reliable velocity information was obtained.

\section{Results}

The results are presented in two sections: firstly the measurements of the particle velocity are considered; and secondly the wall vibrations measured with the laser 


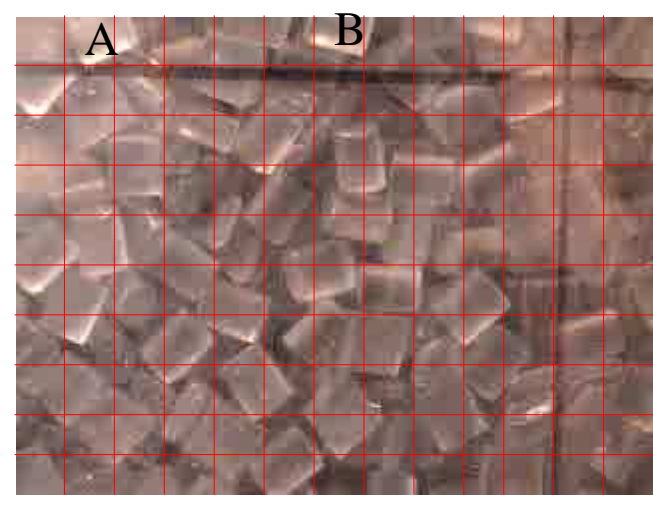

$53 \mathrm{~mm}$

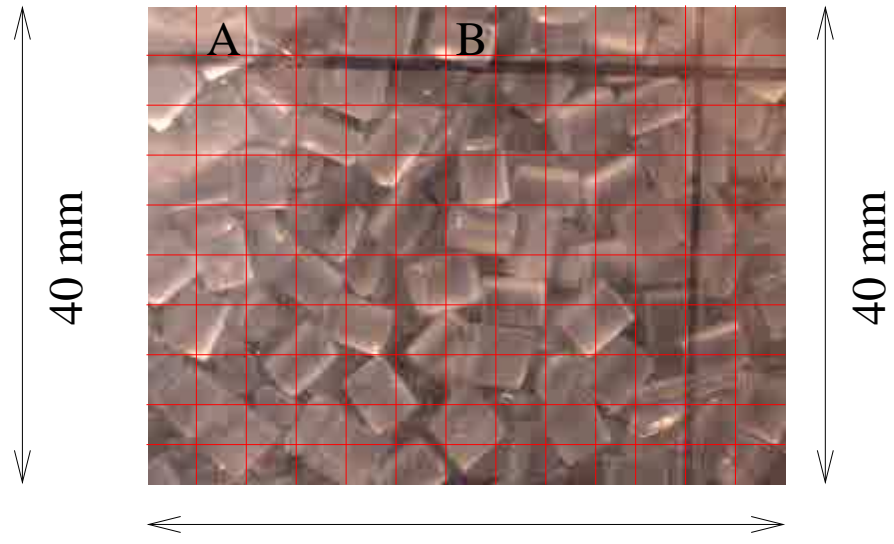

$53 \mathrm{~mm}$

(b) Image 2 , a time $3 \Delta t$ later

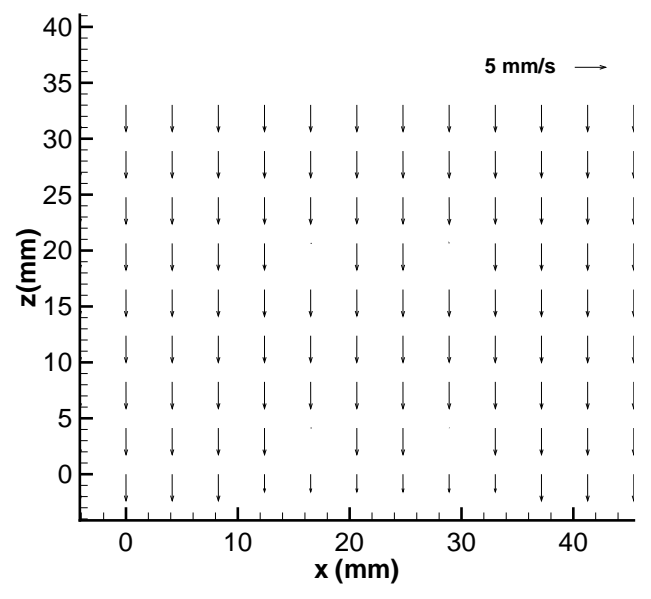

(c) Velocity map

Figure 3. a) Image 1, (b) Image 2 a time $3 \Delta t$ later and (c) the velocity vector map for PET pellets.

vibrometer are discussed.

\subsection{Particle motion on the silo wall}

The following results, unless otherwise stated, correspond to a spatial average over the whole region for which reliable results were obtained. Each set of results is presented with a schematic representing the region of the silo wall at which the original images were recorded.

Figure 5 shows the temporal variation of the particle velocity at the silo wall over a 


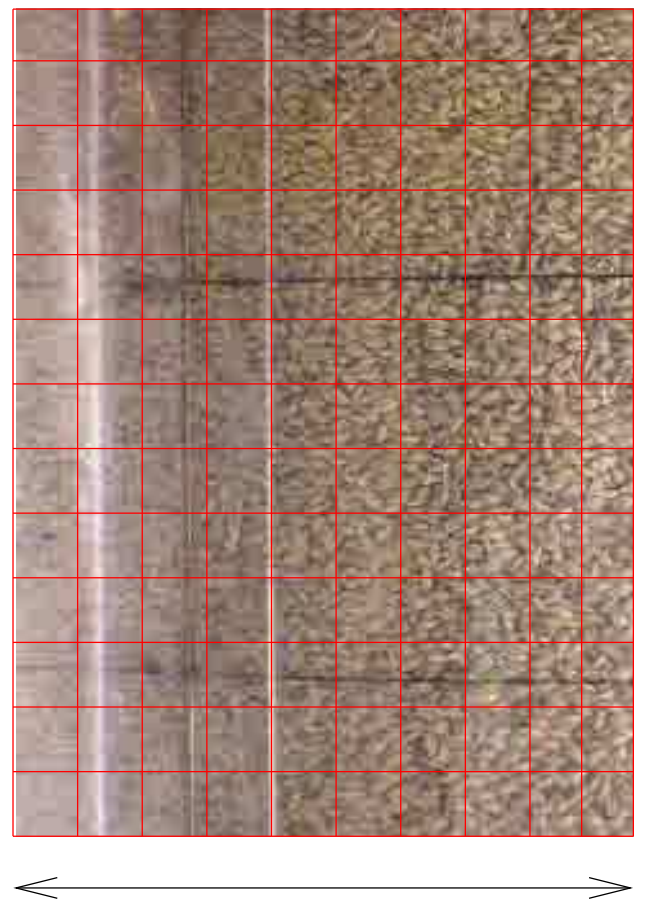

$154 \mathrm{~mm}$

(a) Image 1

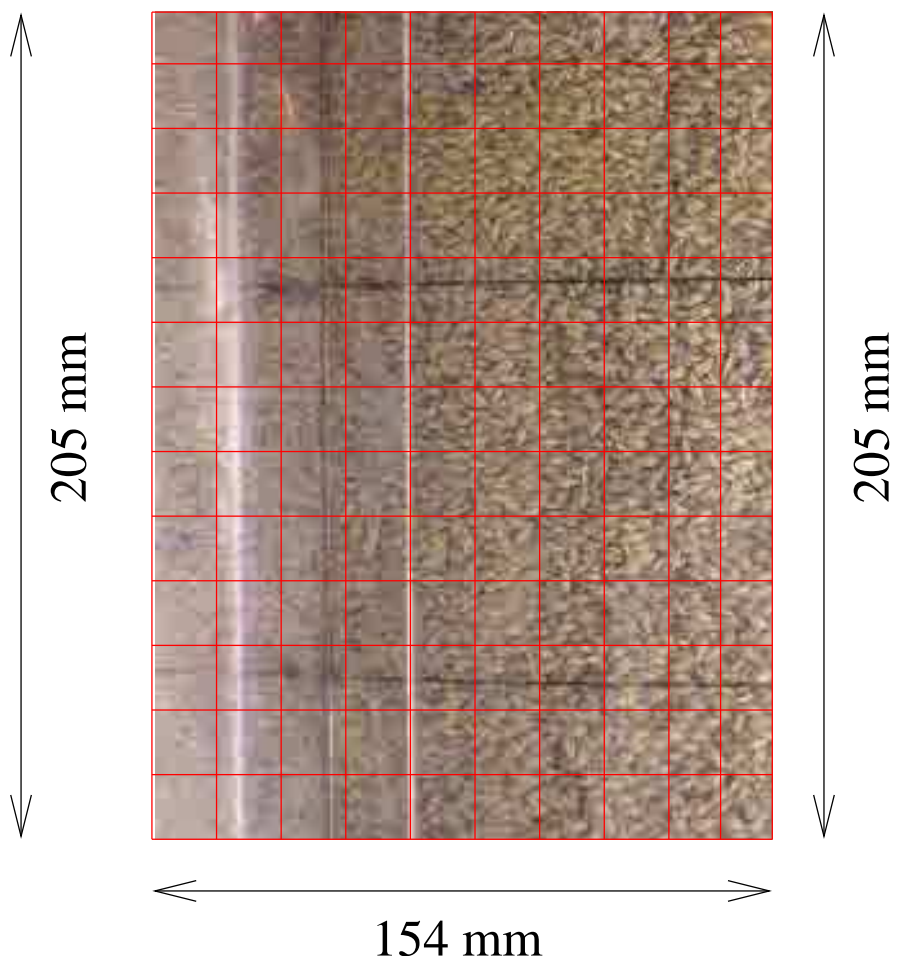

(b) Image 2, a time $\Delta t$ later

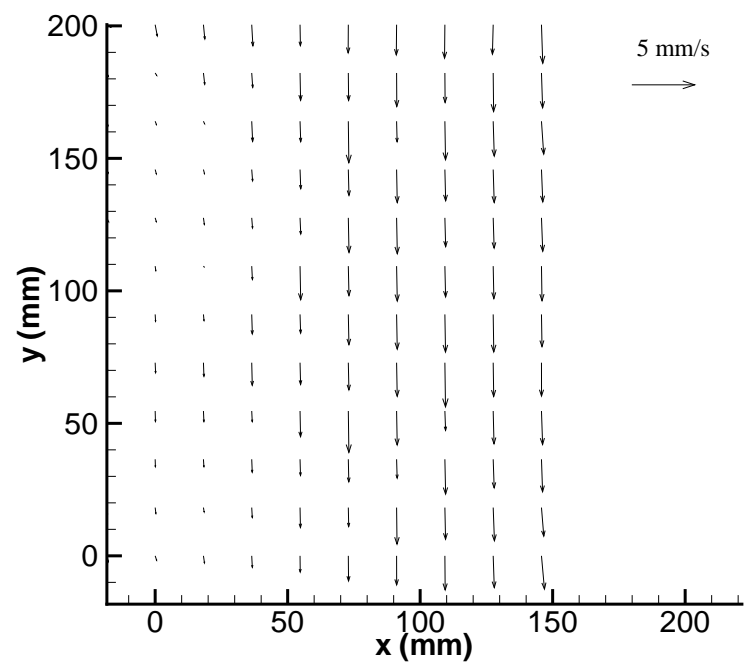

(c) Velocity map

Figure 4. a) Image 1, (b) Image 2 a time $\Delta t$ later and (c) the velocity vector map for barley. 

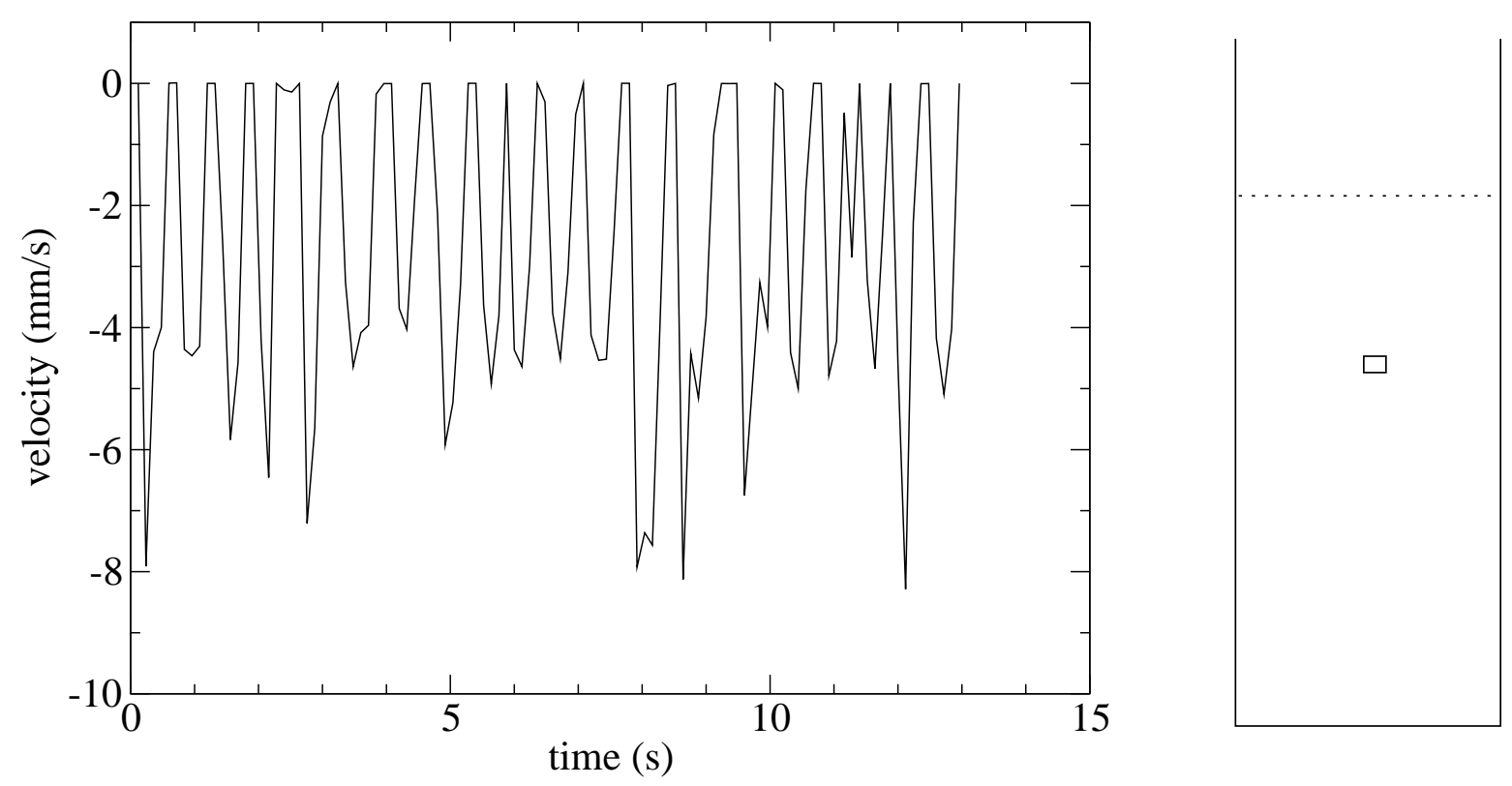

Figure 5. Temporal variation in the spatially averaged velocity for PET pellets. The image to the right of the graph indicates the area of the silo wall where the measurements were taken, the top of the image is approximately $1000 \mathrm{~mm}$ above the base of the silo.

$13 \mathrm{~s}$ time period during the discharge of PET pellets. The results show short periods of the order of a few tens of a second during which the particles are stationary. Between these stationary periods the particles moving along the wall with a downward vertical velocity of the order of 4 to $8 \mathrm{~mm} / \mathrm{s}$. The frequency spectrum of the averaged particle velocity was also calculated and is shown in figure 6 .

As would be expected from the irregular nature of figure 5, the motion does not correspond to a single frequency, however the predominant frequency of the intermittent motion can be seen to be around $1.5 \mathrm{~Hz}$.

Figure 7 shows the spatially averaged velocity for discharging barley. The region over which the image is taken is significantly larger than the region shown in figure 5 . It should be noted that the area marked on the diagram of the silo to the right of the graph in figure 7 corresponds to the area of the full image. Due to problems with reflections on the left-hand side of the image results are only obtained from the right-hand half of the image. Barley exhibits intermittent motion similar to that of PET. The downward velocity in figure 7 appears not to go to zero but shows a slight upward velocity. Further measurements of the barley velocity over smaller regions of the wall confirmed that the velocity of the particles does drop to zero during the "stationary" periods. This suggests that the level of noise introduced by the larger length scale may have caused the slightly upward velocity in figure 7 . The results are also noisier due to the fact that only half the number of values were available to produce the average In general, the velocity of the barley is comparable to the velocity measured for the PET pellets, the main difference between the motion of the two particle types is that the barley remains stationary for 


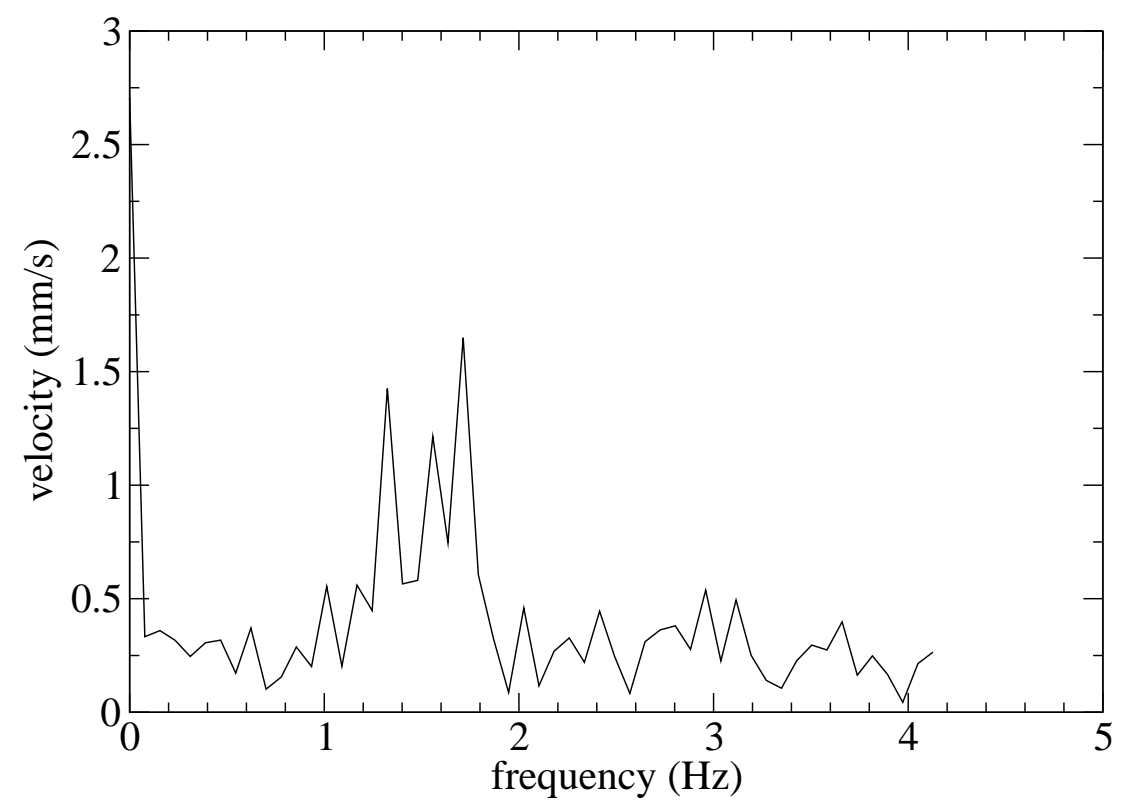

Figure 6. Velocity spectrum during discharge of PET pellets.
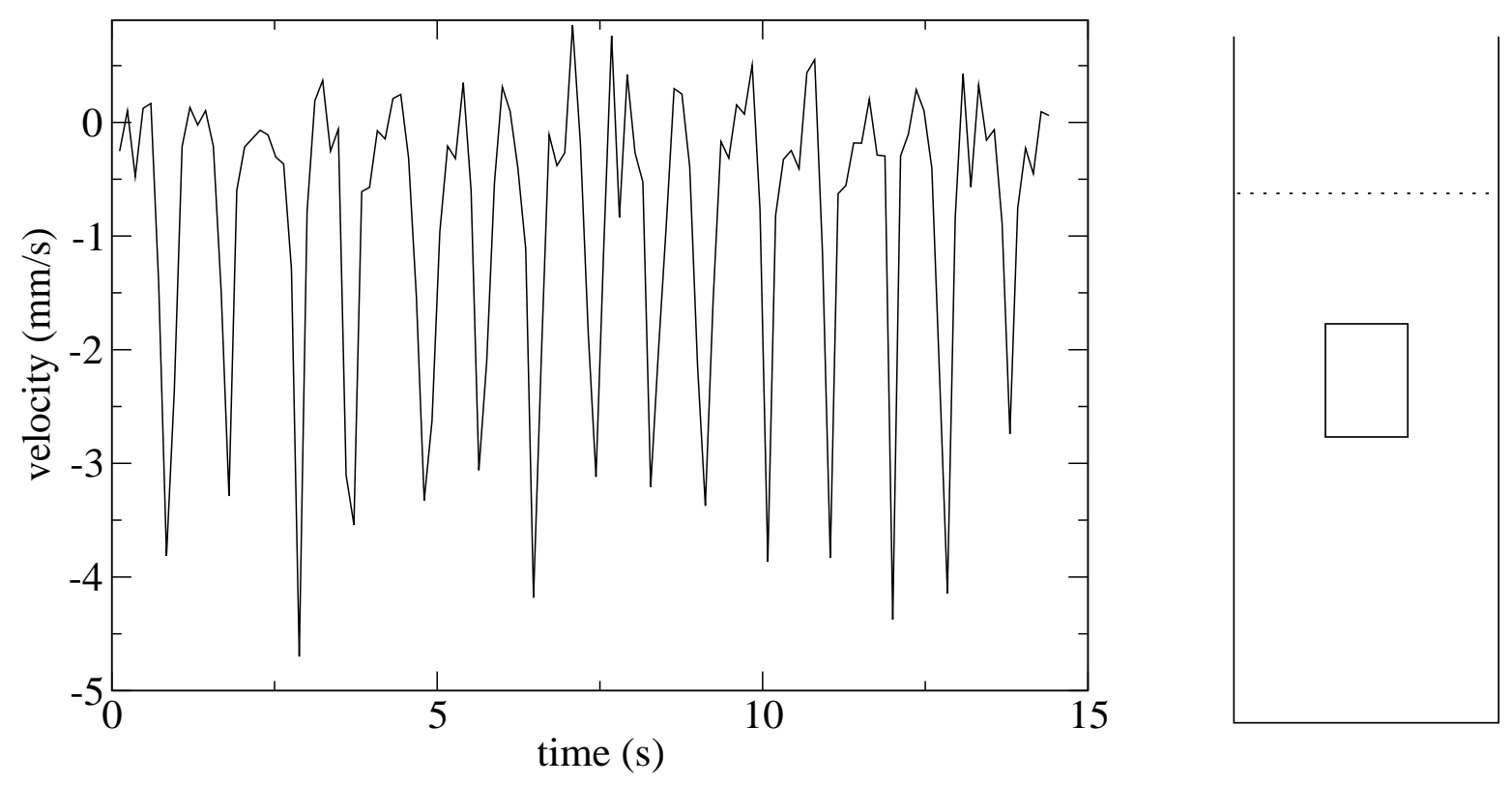

Figure 7. Temporal variation in the spatially averaged velocity for barley.

a longer time. The frequency spectrum for the barley was also calculated and is shown in figure 8. The frequency spectrum shows a more distinct peak than the spectrum for the PET pellets (figure 6) at a frequency slightly greater than $1 \mathrm{~Hz}$.

It is also of interest to determine the temporal mean velocity for both PET and barley particles. This can be found by integrating the velocity profile in figures 5 and 7 


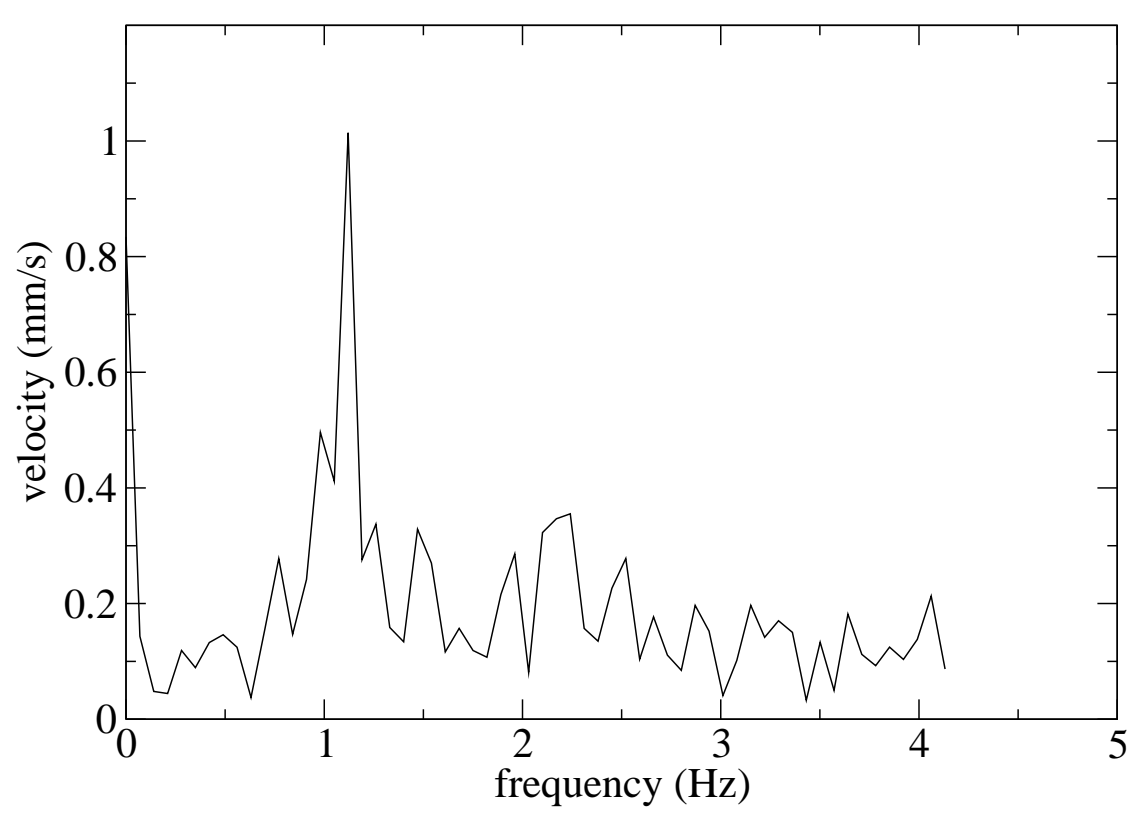

Figure 8. Frequency spectrum for the barley particles against the silo wall.

and was found to be $3.8 \mathrm{~mm} / \mathrm{s}$ and $1.0 \mathrm{~mm} / \mathrm{s}$ respectively. The discharge rate from the model could have been measured during the experiment providing further information but that was not undertaken.

Finally, it is of interest to determine at what length scale the motion of the particles at the wall is in phase. To investigate this, a region of length $500 \mathrm{~mm}$ was imaged with the camera during discharge of barley from the silo. In this case the velocity at a single point (averaged over an interrogation region) was considered as appose to the spatial average over the whole length of the image which are displayed in figures 5 and 7 . This is shown in figure 9a at $z=950 \mathrm{~mm}$ and at the two extremes of the image: $z=700$ $\mathrm{mm}$ and $z=1200 \mathrm{~mm}$. The lower extreme is close to the observed transition region (around $z=600 \mathrm{~mm}$ ) at which the particles stop moving against the wall and the flow becomes internal. The higher extreme is close to the fill height (around $z=1300 \mathrm{~mm}$ ). The results, although significantly noisier than those obtained over a smaller region, still clearly show the start-stop nature of the motion with a frequency slightly greater than $1 \mathrm{~Hz}$. It is also clear that the motion of the particle is very much in phase over a length scale of $0.5 \mathrm{~m}$. This is further examined in figure $9 \mathrm{~b}$ which shows the normalized temporal correlation functions $C_{i j}$, where $i, j \in\{t, m, b\}$ and $t, m$ and $b$ represent the top $(z=1200 \mathrm{~mm})$, middle $(z=950 \mathrm{~mm})$ and bottom $(z=700 \mathrm{~mm})$ respectively. Figure $9 \mathrm{~b}$ shows no significant time lag, $\tau$, between the three signals. The periodic nature of the signals is also evident in figure $9 \mathrm{~b}$ despite the noise. 

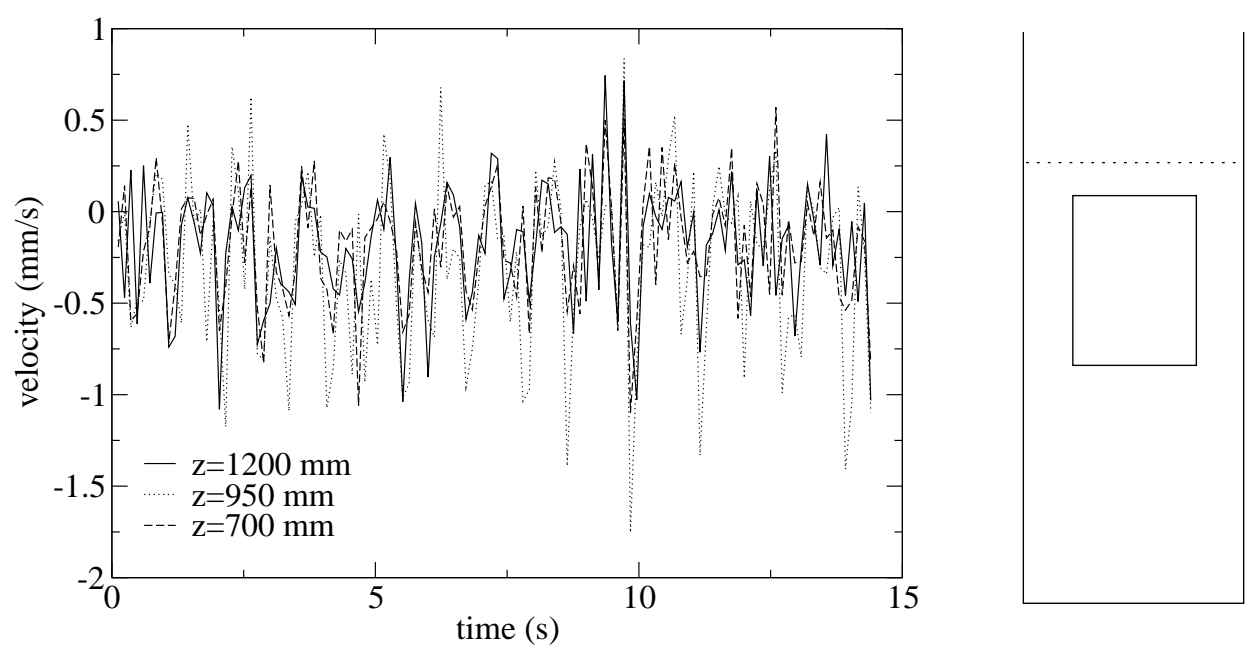

(a) Velocity profile

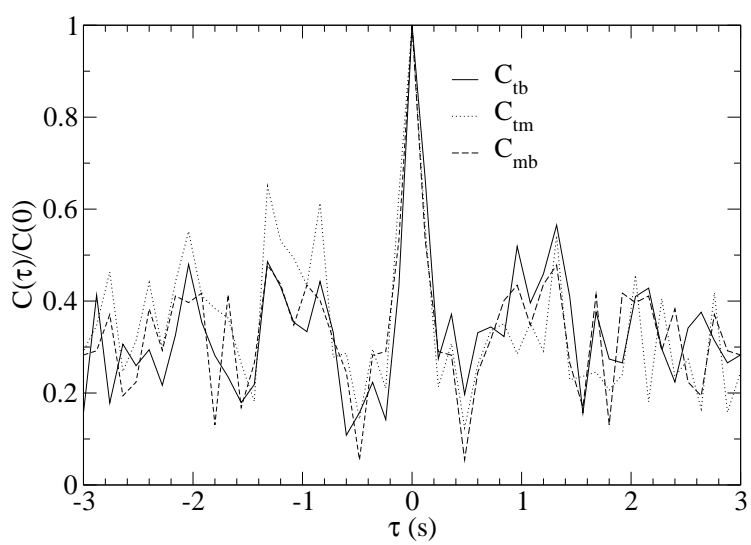

(b) Correlation

Figure 9. Velocity profile of particle motion against the silo wall at three different heights during discharge of barley and the correlations between the heights.

\subsection{Silo wall vibration}

During discharge wall vibrations were observed. These were large enough to be felt by touching the wall. A laser vibrometer was used to measure the radial component of the wall velocity and displacement. Figure 10 shows the velocity and displacement of the walls measured $900 \mathrm{~mm}$ above the base when the silo was filled to $1300 \mathrm{~mm}$ with PET pellet. Figure 10(a) shows the normal displacement and velocity over two selected time intervals. In the first time interval $t=17-20 \mathrm{~s}$, the motion does not appear to be periodic while in the second interval, $t=21-24 \mathrm{~s}$ it does. This difference between the motions during the two time intervals is confirmed by figures 10 (b) and 10(c) which show both the displacement and the velocity spectra for the two time intervals respectively. 
There is a clear peak in figure $10(\mathrm{c})$ at around $3 \mathrm{~Hz}$, corresponding to the periodic motion between $t=21-24 \mathrm{~s}$ but the peak is much smaller in figure 10(b). It is also clear that the motion changes rapidly between $20 \mathrm{~s}$ and $21 \mathrm{~s}$ from non-oscillatory motion observed in figure $10(\mathrm{~b})$ to the oscillatory motion observed in figure $10(\mathrm{c})$. This change, from non-periodic to periodic motion was further investigated. The velocity spectrum as a function of time is presented in figure 11 . The spectrum was calculated using a 2048 point FFT. The sampling rate was 2000 samples per second, each FFT corresponding to approximately $1 \mathrm{~s}$ of data. The time on the axis of figure 11 corresponds to the central time of the FFT. Figure 11 indicates the nature of the intermittent bursts of periodic motion. In each case the peak initially occurs at approximately $3 \mathrm{~Hz}$. There is also evidence of a peak close to $4 \mathrm{~Hz}$; this is particularly evident towards the end of some of the bursts of periodic motion.

Measurements were also obtained when the silo was discharging barley. In this case wall vibrations were also observed, however the intermittent periodic and nonperiodic feature of the vibrations as observed for the PET pellets was not observed for the barley. We also note that the observed velocities were smaller than those observed for the PET particles by an order of magnitude. During discharge of barley, the walls vibrated continuously in a periodic manner as shown in figure 12 . During the discharge of barley the main frequency of vibration is close to $4 \mathrm{~Hz}$, with two smaller peaks at higher frequencies (figure 12).

Multiple measurements were obtained for the peak frequency of the wall vibrations, which were found to be repeatable. The mean value of the peak frequency and the standard deviation were calculated. For the PET particles this was found to be 3.18 $\mathrm{Hz}$ with a standard deviation of $0.133 \mathrm{~Hz}$, while for the barley the mean frequency was $3.89 \mathrm{~Hz}$ with a standard deviation of $0.159 \mathrm{~Hz}$. From these results it is clear that there is a detectable difference in the frequency of the wall vibrations depending on the fill particle.

To investigate this further, the natural frequencies of the model silo were measured. This was done using the laser vibrometer when the silo was filled with PET or barley particles but not discharging. The silo wall was struck radially with a sharp impulse and the response was recorded. For such an impact the frequency response is expected to be dominated by the lowest natural frequency of the silo fill system. The responses are shown in figures 13 and 14 for the PET and barley particles respectively. They show that the free vibration response of the model silo is dependent on the particle fill and that the measured peak frequency observed during discharge corresponds exactly to the natural free vibration frequency of the model silo for both barley and PET.

Finally, it is also of interest to check the ambient vibration response of the silo walls when there is no discharge or applied force on the silo, and vibrations are induced by the surrounding. This was measured for both PET and barley in the silo. In both cases there was a small frequency peak observed at $3 \mathrm{~Hz}$ and $4 \mathrm{~Hz}$ respectively. The magnitude of the peaks were similar for both fills and were an order of magnitude smaller than the measurements for discharging barley and two orders of magnitude smaller than 


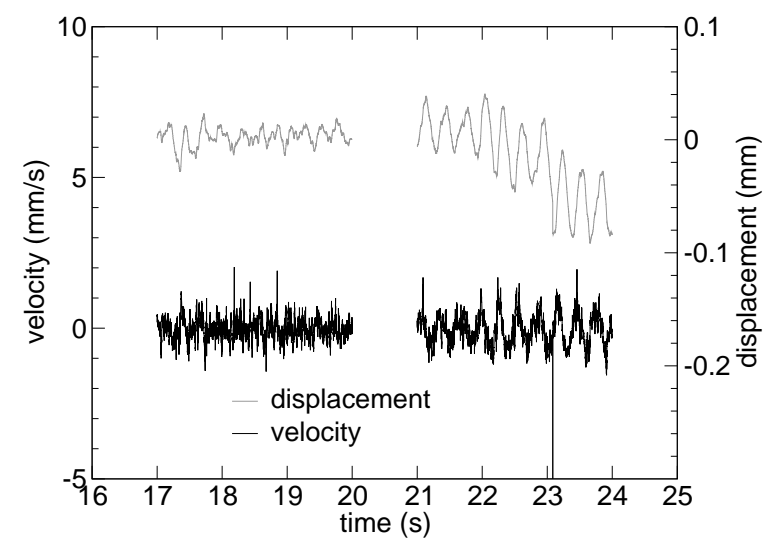

(a) Velocity and displacement

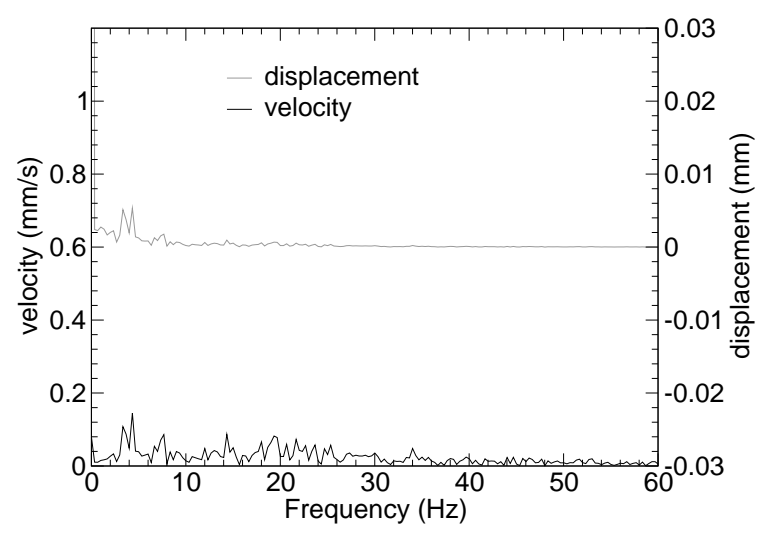

(b) Frequency spectrum for $t=17-20 \mathrm{~s}$

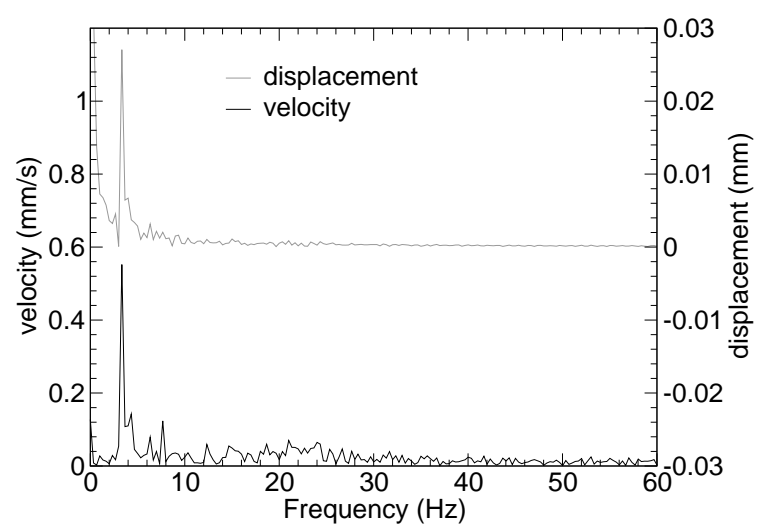

(c) Frequency spectrum for $t=21-24 \mathrm{~s}$

Figure 10. Velocity and displacement measurements during discharge of PET pellets. a) Velocity and displacement at selected times b) Frequency spectrum for $t=17-20 \mathrm{~s}$ period and c) Frequency spectrum for $t=21-24 \mathrm{~s}$ period.

the results for discharging PET. This indicates that when the silo is not discharging, small wall vibrations are induced by external sources. These background vibrations are, however, considerably smaller than the oscillations observed during discharge. The ambient vibration frequencies as well as the oscillation frequencies during discharge closely match the natural frequencies of the model silo-stored solid system.

\section{Discussion}

Experimental studies by the authors $[8,9,13]$ have shown that stick-slip motion of granular solids on a surface is stress dependant and may not be expected to occur in this model silo where the horizontal stresses are expected to be 2 to $3 \mathrm{kPa}$ or smaller. 


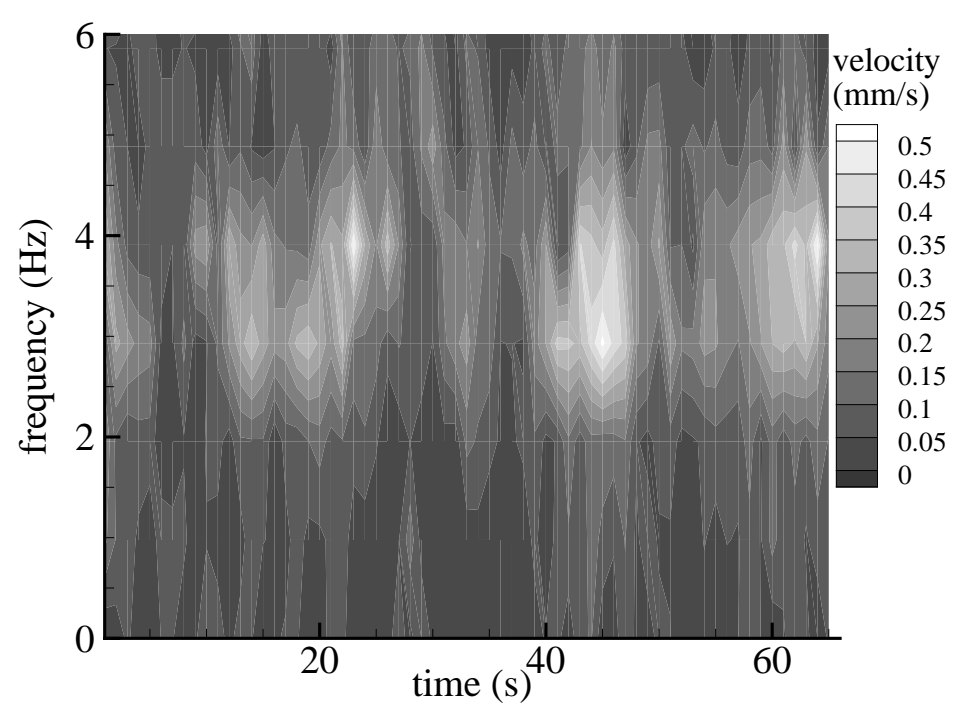

Figure 11. The frequency spectrum as a function of time.

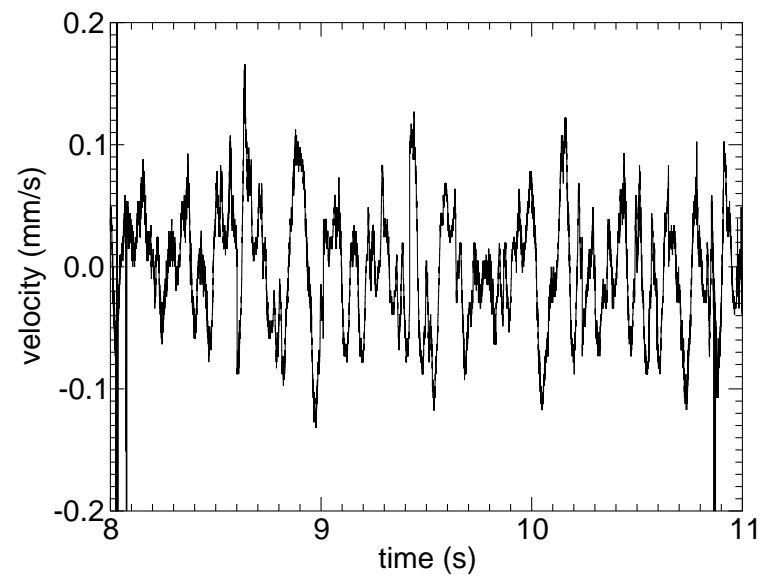

(a)

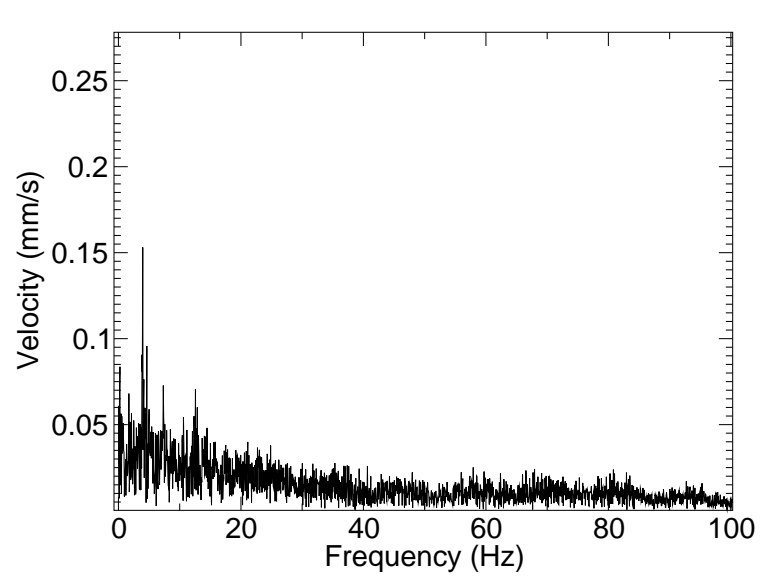

(b)

Figure 12. Velocity measurements during discharge of barley: typical velocity variation with (a) time and (b) frequency. 

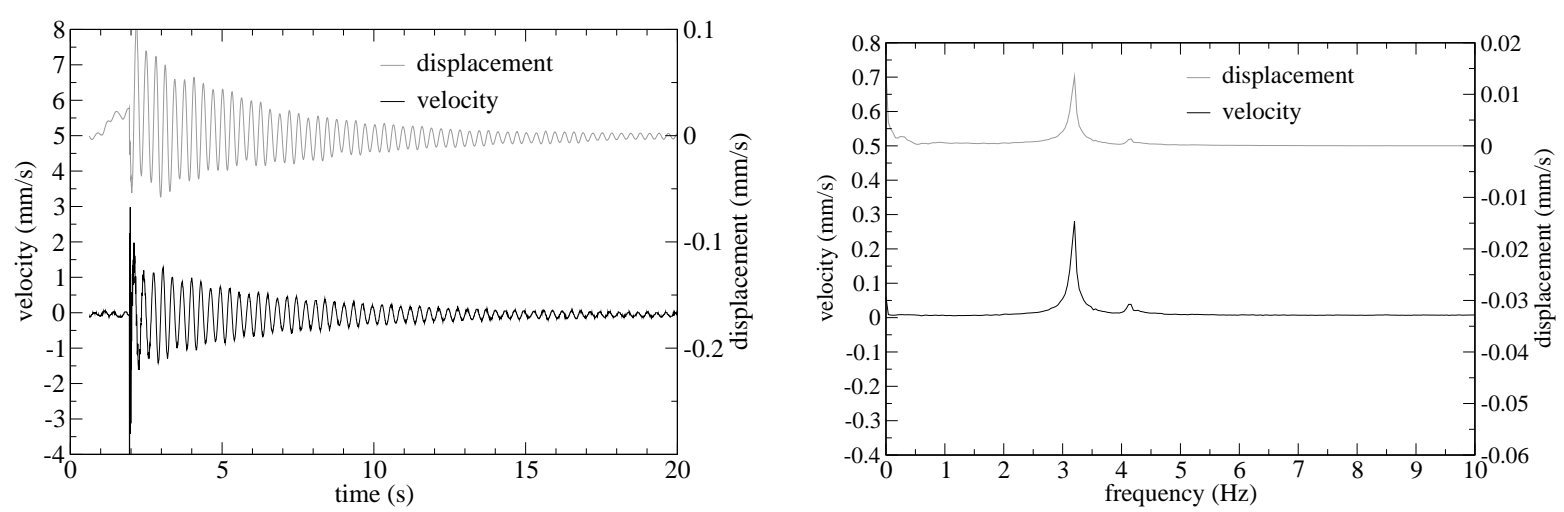

Figure 13. Free vibration of the model silo containing PET pellets.

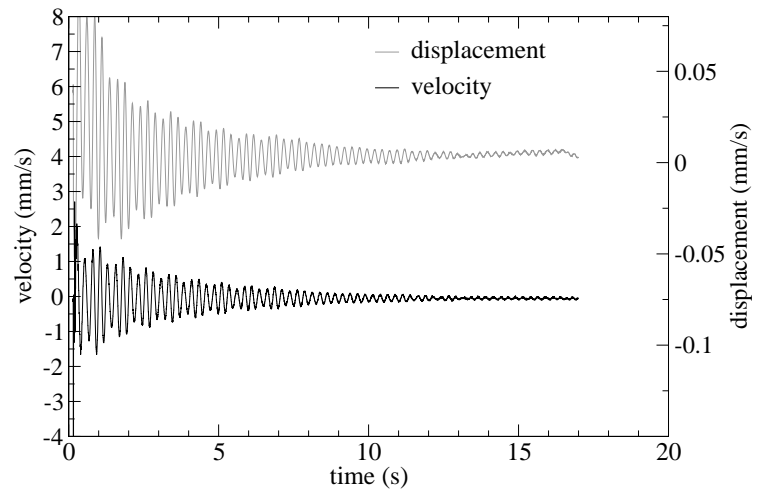

(a)

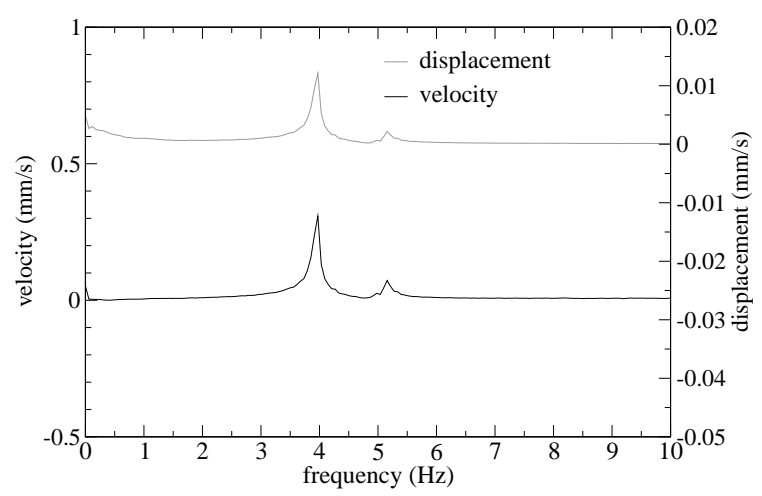

(b)

Figure 14. Free vibration of the model silo containing barley.

Furthermore, whilst stick-slip has been reported for PET pellets on aluminium or steel surfaces, it has not been reported to occur for barley. Thus the intermittent particle motion observed at the silo wall, for both PET and barley could be caused by a different phenomenon and not stick-slip frictional response.

Dynamic effects observed during silo discharge have been discussed earlier. One explanation is that changes in bulk density of the granular medium sets up dilation waves which propagate vertically during discharge, and that the observed frequency of the intermittent particle relates to this wave propagation. Further work is needed to verify if this can offer a plausible explanation.

What is however incontrovertible is that the dynamic excitation caused by the silo discharge has set the model silo vibrating with its lowest natural free vibration frequency (3.9 Hz for the barley-silo system and $3.2 \mathrm{~Hz}$ for the PET-silo system) rather than the frequency of the excitation. For both types of fill particle, the frequency of the time- 
variations of the particle velocities near the wall are significantly lower than the resulting wall vibrations (by a factor of 2 and 4 for the PET and barley respectively). It might be expected that coupling at the silo wall would induce vibrations with the same frequency as the particle velocities, however, such vibrations are not supported in the particle-silo system. Rather the particle-silo system vibrates at its natural frequency. Silo honking has already been shown by the authors [8,9] and others [7] to exhibit a much higher frequency than the lower natural frequencies of the silo-solid system. Thus the vibration response of this model silo is more akin to the silo quaking/shaking rather than honking. The mechanism that leads to silo honking appears not to have manifested itself here.

It is also worth noting that the magnitude of wall vibrations for PET is an order of magnitude greater than that for barley. This can be attributed to several factors including the difference in properties of the two materials and their interaction with the silo wall. It indicates that PET can generate significantly larger vibrations in silos and is thus more prone to causing undesirable dynamic effects on the silo structure than barley. PET pellets are known to produce honking and quaking in industrial silos and also exhibit strong stick-slip motion in laboratory friction tests under stress levels pertaining to full-scale situations $[9,13]$. Barley has neither been reported to cause silo honking nor been found to exhibit stick-slip motion in laboratory friction tests.

\section{Conclusions}

A study of the particle motion and associated wall vibration during discharge of a model silo has been presented. The motion of the particles against the silo wall has been observed to be intermittent fashion. This motion was measured quantitatively giving a record of the particle velocity as a function of time. The frequency spectra of the intermittent motion were evaluated for the two fill particles: PET and barley. For both fills, the particles were seen to remain stationary at the wall for a time of the order of a tenth of a second and then slide down the side of the wall before returning to rest. The time during which the particles were in motion was several times larger than their stationary period with an overall repetition frequency of between 1 and $2 \mathrm{~Hz}$. The particles were also observed to be moving in phase over the entire silo wall (above the transition level). The observed motion cannot be readily linked to the natural frequency of the wave propagation in the solid.

Horizontal wall vibrations were observed and measured during discharge. In general the radial vibration response was periodic, though typical durations of non-periodic motion were observed during discharge of PET pellets. The oscillatory motion was found to contain a single peak frequency which corresponded to the natural frequency of free vibration of the full silo. The discharge dynamics has caused the silo to vibrate in its natural free vibration frequencies of $3.9 \mathrm{~Hz}$ for barley and $3.2 \mathrm{~Hz}$ for PET. The vibration response is thus more akin to silo quaking/shaking than honking. 


\section{Acknowledgements}

The authors would like to acknowledge the financial and practical assistance of Braby Silos; Dow Chemical Company; DuPont; DuPont Teijin Films; Eastman Chemical Company; Jansens\& Dieperink; Jenike \& Johanson Inc.; and Waeschle and the financial support of EPSRC UK (grant no. GR/R44263) and an ORS Award (ChavezSagarnaga). The authors would also like to thank C. A. Greated, D. M. Campbell and J. A. Cosgrove for useful discussions.

\section{References}

[1] Roberts AW and Wiche SJ 1991 Silo quaking - a pulsating load problem during discharge in bins and silos (Proc., Bulk 2000 Conference, IMechE, London) 7-12.

[2] Gudehus G and Tejchman J. 1992 Silo music and silo quake (Silos - Forschung und Praxis Tagung '92, Karlsruhe, October) 103-10.

[3] Roberts A W 1993 Mechanics of self excited dynamic loads in bins and silos (Proc. Int. Symp. Reliable Flow of Particulate Solids II, Oslo) 983-1004.

[4] Tejchman J 1995 Silo quake - experiments and a polar hypoplastic model (European Symp: Storage and Flow of Particulate Solids (Janssen Centennial), March, Nurnberg) 151-62.

[5] Schulze D 1998 Silo Quaking in Silos - Fundamentals of theory, behaviour and design (eds Brown C J and Nielsen J, E \& FN Spon, London) 171-82.

[6] Ooi JY, Zhong Z and Hardy M S H 1999 Investigation of vibration and honking in DuPont Silos (Confidential Technical Investigation Report, University of Edinburgh) 30pp.

[7] Tejchman J 1999 Powder Technology 106 70-22.

[8] Buick J M, Chavez-Sagarnaga J, Zhong Z, Ooi J Y, Pankaj, Campbell D M and Greated C A 2003 Investigation of silo honking: slip-stick excitation and wall vibration (16th ASCE Engineering Mechanics Conference, Seattle, paper 625).

[9] Buick J M, Chavez-Sagarnaga J, Zhong Z, Ooi J Y, Pankaj, Campbell D M and Greated C A 2003b Investigation of silo honking: slip-stick excitation and wall vibration, submitted to $J$. Eng. Mech. ASCE.

[10] Tejchman J and Gudehus G 1993 Powder Technology 76 201-12.

[11] Muite B K, Shandon F, Quinn S F, Sundaresan S and Rao K K 2004 Silo music and silo quake: granular flow induced vibrations. Submitted to Powder Technology. http://arxiv.org/ftp/condmat/papers/0310/0310086.pdf

[12] Wensrich C 2002 Powder Technology 127 87-94.

[13] Chavez-Sagarnaga J, Buick J M, Ooi J Y, Pankaj, Campbell D M, Greated C A 2004 Frictional properties of pellets and silo wall materials for the investigation of silo honking (Proc., Int. 
Congress for Particle Technology, PARTEC 2004, Nuremberg, Germany, March 2004) 4pp.

[14] Adrian R J 1991 Ann. Rev. Fluid Mech 23 261-304.

[15] Raffel M, Willert C and Kompenhans J. 1998 Particle Image Velocimetry: A Practical Guide (Springer, Heidelberg).

[16] Buick J M, Cosgrove J A, Campbell D M and Greated C A 2002 Development of an optical measuring technique for the study of acoustical phenomena, in Optical Methods and Data Processing in Heat and Fluid Flow (Professional Engineering Publishing) 133-42.

[17] Bishop R E D and Johnson D C 1956 Vibration Analysis Tables (Cambridge University Press).

[18] Rotter J M 2000 Guide for the economic design of circular metal silos (Spon Press, London).

[19] AS 37741990 Loads on Bulk Solids Containers (Australian Standards with Commentary, Standards Association of Australia, Sydney) 


\section{Figure captions}

Figure 1. Experimental set-up.

Figure 2. Analysis of the digital images to obtain velocity information.

Figure 3(a): Image 1

Figure 3(b): Image 2, a time $3 \Delta t$ later

Figure 3(c): Velocity map

Figure 3. a) Image 1, (b) Image 2 a time $3 \Delta t$ later and (c) the velocity vector map for PET pellets.

Figure 4(a): Image 1

Figure 4(b): Image 2, a time $\Delta t$ later

Figure 4(c): Velocity map

Figure 4. a) Image 1, (b) Image 2 a time $\Delta t$ later and (c) the velocity vector map for barley.

Figure 5. Temporal variation in the spatially averaged velocity for PET pellets. The image to the right of the graph indicates the area of the silo wall where the measurements were taken, the top of the image is approximately $1000 \mathrm{~mm}$ above the base of the silo.

Figure 6. Velocity spectrum during discharge of PET pellets. 
Figure 7. Temporal variation in the spatially averaged velocity for barley.

Figure 8. Frequency spectrum for the barley particles against the silo wall.

Figure 9(a): Velocity profile

Figure 9(b): VCorrelation

Figure 9. Velocity profile of particle motion against the silo wall at three different heights during discharge of barley and the correlations between the heights.

Figure 10(a): Velocity and displacement

Figure 10(b): Frequency spectrum for $t=17-20 \mathrm{~s}$

Figure 10(c): Frequencu spectrum for $t=21-24 \mathrm{~s}$

Figure 10. Velocity and displacement measurements during discharge of PET pellets. a) Velocity and displacement at selected times b) Frequency spectrum for $t=17-20 \mathrm{~s}$ period and c) Frequency spectrum for $t=21-24 \mathrm{~s}$ period.

Figure 11. The frequency spectrum as a function of time.

Figure 12. Velocity measurements during discharge of barley: typical velocity variation with (a) time and (b) frequency.

Figure 13. Free vibration of the model silo containing PET pellets.

Figure 14. Free vibration of the model silo containing barley. 